\title{
Drug rash with eosinophilia and systemic symptoms and graft-versus-host disease developing sequentially in a patient
}

\author{
J. Natkunarajah, K. Watson, S. Diaz-Cano,* G. Mufti,† A. du Vivier and D. Creamer
}

Departments of Dermatology, ${ }^{*}$ Histopathology and +Haematological Medicine, King's College Hospital, London, UK

Summary

We describe a case of drug rash with eosinophilia and systemic symptoms (DRESS) and graft-versus-host disease (GvHD) developing sequentially in a patient displaying reactivation of CMV. We discuss the possibility that similar pathogenic mechanisms may be involved in the development of DRESS and GvHD.

Drug rash with eosinophilia and systemic symptoms (DRESS) is a severe acute drug reaction characterized by a polymorphic skin eruption associated with fever, eosinophilia, lymphadenopathy and multiorgan involvement. ${ }^{1}$ The development of DRESS has been associated with reactivation of herpesviruses. ${ }^{2,3}$ This relationship is similar to the relationship between herpesvirus reactivation and the development of graftversus-host disease (GvHD). ${ }^{4}$ We report a case of DRESS and GvHD developing sequentially in a patient displaying reactivation of cytomegalovirus (CMV).

\section{Report}

A 62-year-old white man with follicular non-Hodgkin's lymphoma underwent an allogeneic haemopoietic stemcell transplant (HSCT) from a matched unrelated donor, after preparation with alemtazumab and fludarabine treatment. After the transplant, the patient received ciclosporin for GvHD prophylaxis and aciclovir for prophylaxis against CMV reactivation. There was evidence of previous CMV infection; however, this was quiescent at the time of the transplant (pretransplant CMV DNA load $<10$ copies $/ \mathrm{mL}$ ). The donor was CMV-positive.

On day 18 post-HSCT, the patient became unwell with a Gram-negative bacteraemia and was started on tazocin, gentamicin, vancomycin and meropenem.

Correspondence: Dr Janakan Natkunarajah, Department of Dermatology, Amersham Hospital, Whielden Street, Amersham, HP7 0JD, UK.

E-mail: janakan@fsmail.net

Conflict of interest: none declared.

Accepted for publication 14 December 2007
Three days later he developed a widespread, pruritic, confluent erythema on the face, trunk and arms. There was no mucosal involvement and the palms and soles were spared.

Histology showed a spongiotic dermatitis with focal parakeratosis, vesicle formation and prominent extravasated red blood cells (Fig. 1a). There was evidence of
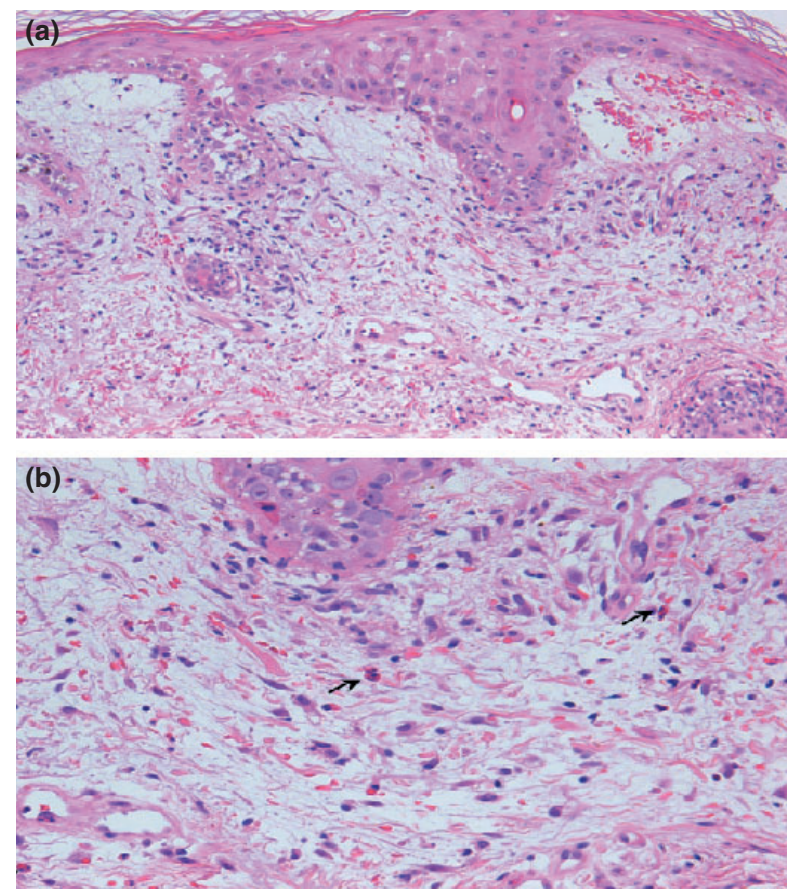

Figure 1 (a) Spongiotic dermatitis with focal parakeratosis and vesicle formation, with prominent extravasated red blood cells; (b) occasional necrotic keratinocytes without satellitosis. Haematoxylin and eosin, original magnification $(a, b) \times 100$. 

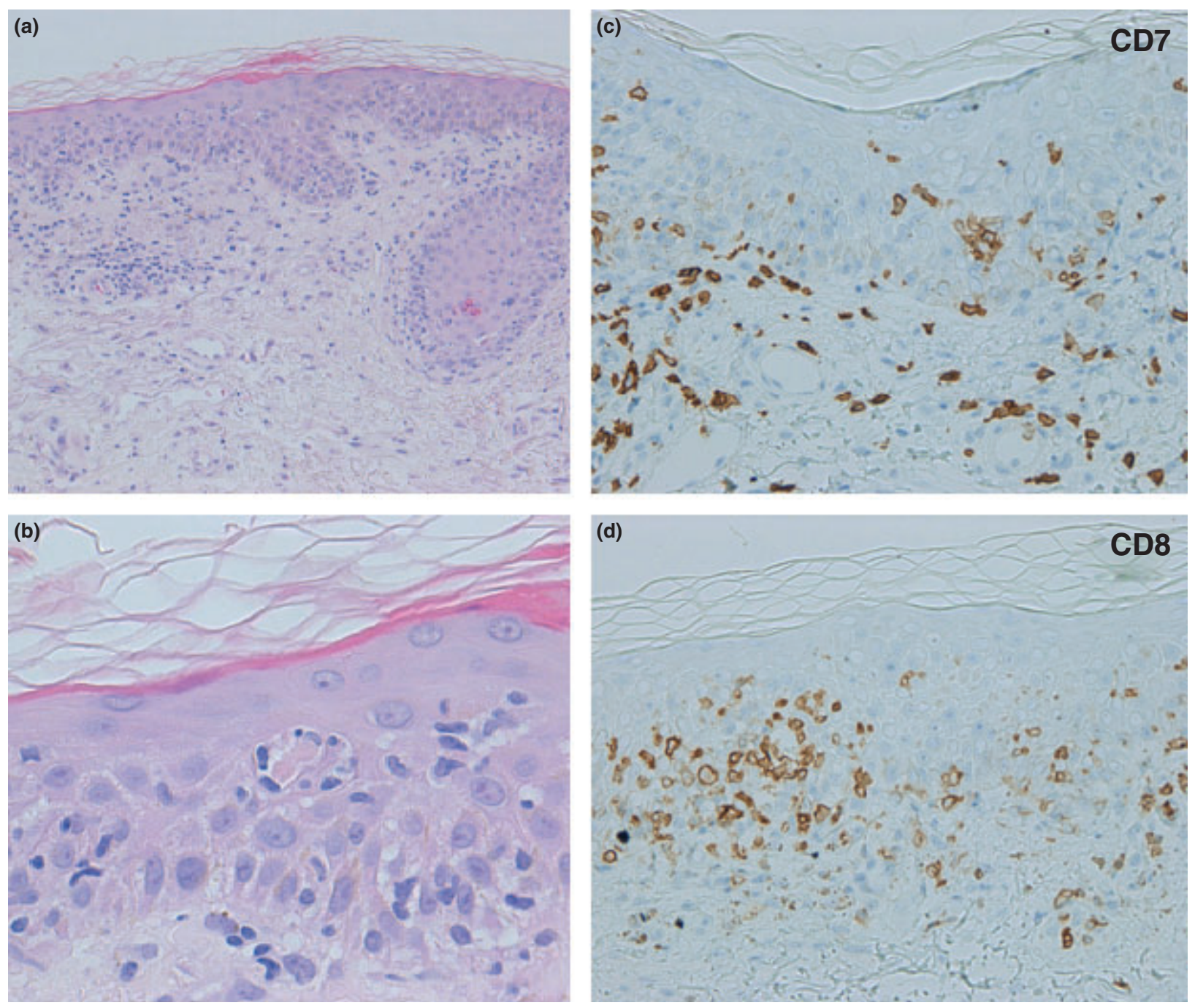

Figure 2 Graft-vs.-host disease. (a) Vacuolar interface dermatitis with focal parakeratosis, spongiosis and lymphocyte exocytosis (haematoxylin and eosin, original magnification $\times 400$ ); (b) Spongiotic epidermis with necrotic keratinocyte and lymphocyte satellitosis (haematoxylin and eosin, original magnification $\times 400)$; (c) Intraepidermal lymphocytes demonstrate mature $(\mathrm{CD} 7+)$ immunophenotype; $(\mathrm{d})$ Intraepidermal lymphocytes dsemonstrate cytotoxic (CD8+) immunophenotype (immunostaining, original magnification $\times 400$ ).

occasional necrotic keratinocytes without satellitosis with a chronic inflammatory cell infiltrate that included eosinophils (Fig. 1b, arrowed). There were no histological features of GvHD.

A drug-induced exanthema was diagnosed and vancomycin was discontinued as it was thought to be the causative agent. However, 19 days later the patient remained unwell with fever, erythroderma, marked oedema of the face and limbs and widespread lymphadenopathy. At this stage all antibiotics were discontinued.

Laboratory investigations showed eosinophilia of $6.9 \times 10^{9} / \mathrm{L}$ (normal range $0.0-0.4 \times 10^{9} / \mathrm{L}$ ) and abnormal liver-function results, with a raised alkaline phosphatase of $170 \mathrm{IU} / \mathrm{L}(30-130)$ and $\gamma$-glutamyl transpeptidase $183 \mathrm{IU} / \mathrm{L}(1-55)$.

A diagnosis of DRESS was made, and the patient was treated with prednisolone $40 \mathrm{mg}$ daily and a potent topical corticosteroid ointment. The erythema, oedema, eosinophilia and liver-function abnormalities improved over the next 3 weeks. Further tests performed at this time showed evidence of recent $\mathrm{CMV}$ reactivation (CMV DNA 2687 copies $/ \mathrm{mL}$ ). The prednisolone was tapered and discontinued and the patient was started on vanciclovir $450 \mathrm{mg}$ daily.

Seven weeks after the first eruption (day 71 postHSCT), the patient developed a new exanthema with a 
pruritic erythematous macular eruption involving the trunk and a confluent erythema on the dorsal surfaces of the hands and feet. Histology revealed prominent lymphocyte exocytosis with basal vacuolation and satellite-cell necrosis (Fig. 2a,b). The intraepidermal lymphocytes showed a mature $(\mathrm{CD} 7+)$ (Fig. 2d) and cytotoxic (CD8+) (Fig. 2c) immunophenotype. These features were indicative of GvHD. The patient was again treated with prednisolone, resulting in rapid clearance of the dermatosis.

We report an HSCT recipient who underwent CMV reactivation with development of DRESS initially and then acute GvHD. It is recognized that infection with, or reactivation of, herpesviruses following HSCT is associated with a graft-versus-host reaction. ${ }^{4}$ Maeda et al. showed a temporal relationship between detection of DNA of certain herpesviruses and appearance of GvHD. ${ }^{5}$ In Maeda's study, human herpesvirus 6 was identified early (3-4 weeks post-transplant), whereas CMV and EBV were detected at 2 months and 3 months posttransplant, respectively, and coincided with the development of GvHD. ${ }^{5}$ The role of CMV specifically in the induction of GvHD has been suggested by studies showing that human leucocyte antigen (HLA)-DR7restricted CMV-specific CD4 T lymphocytes can display specificity to the alloantigen HLA-DR $4 .{ }^{6}$

DRESS is a severe acute drug reaction characterized by a polymorphic skin eruption associated with fever, eosinophilia, lymphadenopathy and multiorgan involvement. ${ }^{1}$ Sulfonamide and anticonvulsants are most often implicated as causal agents in DRESS, but vancomycin is also a known drug culprit. ${ }^{1,7}$ The development of DRESS has been associated with reactivation of $\mathrm{CMV}^{2}$ as well as other members of the herpesvirus family. ${ }^{3}$ In a recent issue of the British Journal of Dermatology, Seishima et al. ${ }^{8}$ noted positive CMV DNA and an increased CMV IgG antibody titre in all seven patients with DRESS and also showed a high frequency of HHV6 and HHV7 positivity in this group of patients. In the same issue Kano et al. ${ }^{3}$ showed a sequential pattern of herpesvirus reactivation in four patients with DRESS, in which HHV6 and EBV were reactivated early, followed by HHV7 and finally CMV. This sequential cascade mirrors the pattern of virus reactivation that has been described after bone-marrow transplantation and HSCT. ${ }^{9}$ Kano et al. ${ }^{3}$ were also able to demonstrate a relationship between various clinical features of DRESS and reactivation of the different human herpesviruses. These observations have led researchers to believe that reactivation of human herpesviruses is pathogenetic in the development of both DRESS and GvHD, and that a sequential pattern of viral expression is a common element in the pathways of both disorders. However, we cannot exclude the possibility that other herpesviruses may have played a role in the development of both DRESS and GvHD in our case, as titres of other herpesviruses were not measured.

Kano et al. ${ }^{10}$ were first to report a case of sclerodermoid GvHD after resolution of DRESS; however, it remains unknown whether the herpesviruses were the aetiological trigger. We describe another case of DRESS and GvHD occurring consecutively in a patient who displayed reactivation of CMV. We speculate that the CMV upregulation may have had a role in triggering both conditions in this individual.

\section{References}

1 Sullivan JR, Shear NH. The drug hypersensitivity syndrome. Arch Dermatol 2001; 137: 357-64.

2 Aihara M, Sugita Y, Nagatani T et al. Anticonvulsant hypersensitivity syndrome associated with reactivation of cytomegalovirus. Br J Dermatol 2001; 144: 1231-4.

3 Kano Y, Hiraharas K, Sakuma K et al. Several herpesviruses can reactivate in a severe drug-induced multiorgan reaction in the same sequential order as in graft-versushost disease. Br J Dermatol 2006; 155: 301-6.

4 Gratama JW, Middeldorp JM, Sinnige LG et al. Cytomegalovirus immunity in allogeneic marrow grafting. Transplantation 1985; 40: 510-4.

5 Maeda Y, Teshima T, Yamada M et al. Monitoring of human herpesviruses after allogeneic peripheral stem cell transplantation and bone marrow transplantation. $\mathrm{Br} \mathrm{J}$ Haematol 1999; 105: 295-302.

6 Elkington R, Khanna R. Cross-recognition of human alloantigen by cytomegalovirus glycoprotein-specific CD4+ cytotoxic T lymphocytes: implications for graft-versus-host disease. Blood 2005; 105: 1362-4.

7 Zuliani E, Zwahlen H, Gilliet F, Marone C. Vancomycininduced hypersensitivity reaction with acute renal failure: resolution following cyclosporine treatment. Clin Nephrol 2005; 64: 155-8.

8 Seishima M, Yamanaka S, Fijisawa T et al. Reactivation of human herpesvirus (HHV) family members other than HHV-6 in drug-induced hypersensitivity syndrome. $\mathrm{Br} \mathrm{J}$ Dermatol 2006; 155: 344-9.

9 Maeda T, Teshima T, Yamada M, Marada M. Reactivation of human herpesviruses after allogeneic peripheral blood stem cell transplantation and bone marrow transplantation. Leuk Lymphoma 2000; 39: 229-39.

10 Kano Y, Sakuma K, Shiohara T. Sclerodermoid graft-versus-host disease-like lesions occurring after drug-induced hypersensitivity syndrome. Br J Dermatol 2007; 156: 1061-3. 